\title{
Plasmonic Hydrogen Sensor at Infrared Wavelengths
}

\author{
Yoshiaki Nishijima, ${ }^{*}$ Armandas Balčytis, ${ }^{1,2}$ Gediminas Seniutinas, ${ }^{1,3}$ \\ Saulius Juodkazis, ${ }^{1}$ Taro Arakawa, Shinji Okazaki, ${ }^{4}$ and Raimondas Petruškevičius ${ }^{2}$ \\ Department of Electrical and Computer Engineering, Graduate School of Engineering, \\ Yokohama National University, 79-5 Tokiwadai, Hodogaya-ku, Yokohama, Kanagawa 240-8501, Japan \\ ${ }^{1}$ Centre for Micro-Photonics, Faculty of Engineering and Industrial Sciences, \\ Swinburne University of Technology, Hawthorn, VIC 3122, Australia \\ ${ }^{2}$ Institute of Physics, Center for Physical Sciences and Technology, \\ 231 Savanori1269u Avenue, LT-02300 Vilnius, Lithuania \\ ${ }^{3}$ Paul Scherrer Institute, Villigen CH-5232, Switzerland \\ ${ }^{4}$ Department of Materials Science and Engineering, Graduate School of Engineering, \\ Yokohama National University, 79-5 Tokiwadai, Hodogaya-ku, Yokohama, Kanagawa 240-8501, Japan
}

(Received February 14, 2017; accepted June 7, 2017)

Keywords: plasmon sensor, hydrogen gas detection, infrared spectroscopy

We have investigated the variations in the transmission spectra of nanodiscs as well as metal hole array plasmonic structures as a result of the hydrogen adsorption-desorption processes. The structures have been shown to be a viable platform for all-optical hydrogen sensing in the infrared. In cases of both nanodisc and metal hole arrays, upon exposure to hydrogen, a redshift of the plasmon resonance band was observed. Response time as well as the magnitude of the spectral shift was similar for both plasmonic structure types.

\section{Introduction}

Following recent advances in the development of fuel-cell powered vehicles as well as the push towards a hydrogen-based energy economy, the demand for hydrogen sensors continues to rise. ${ }^{(1)}$ In light of the explosive volatility of hydrogen-air mixtures, mitigation of risk will become an ever more pressing safety concern. For this purpose, optical sensors provide a more secure approach for hydrogen leak detection than their electrical counterparts. ${ }^{(2,3)}$ A vigorously pursued direction involves monitoring hydrogen-induced optical changes in palladium, either directly ${ }^{(4-7)}$ or indirectly, using gold nanoparticles as optical transducers. ${ }^{(8)}$ Another important direction in such plasmonic hydrogen detection involves investigating how metal properties can be optimized through alloying. Our previous work demonstrated how the hydrogen-induced optical response of pure palladium compares with its noble metal alloys. ${ }^{(10)}$ It was shown that alloying Pd and $\mathrm{Au}$ increases the rate of $\mathrm{H}_{2}$ accommodation/extraction reactions. However, as will be discussed further, plasmonic oscillations in Pd experience significant damping at visible wavelengths. Therefore, the mid-infrared spectral region is more amenable for direct optical readout. In this study, we demonstrate how the mid-infrared transmittance properties of Pd nanodisc array (NDA) and metal hole array (MHA) plasmonic structures change in the presence of hydrogen. NDA and MHA are the basic shapes of plasmonic materials, but the optical response is inverted to each other

*Corresponding author: e-mail: nishijima-yoshiaki-sp@ynu.ac.jp

http://dx.doi.org/10.18494/SAM.2017.1581 
(NDA, reflection or scattering; MHA, transmission). Therefore, it is important to reveal the optical response of the hydrogen storage process of both NDA and MHA, to construct the plasmonic hydrogen sensors.

\section{Samples and Methods}

NDA and MHA nanostructures were fabricated on a double-side-polished 8 inch silicon wafer via reduction projection photolithography techniques using an $i$-line stepper (NSR205-i14E, NIKON Corporation). The same photomask pattern was used for both NDA and MHA fabrication; however, for obtaining NDAs, positive-tone photoresists, (TLOR-P003 HP, Tokyo Ohka Kogyo Co., Ltd.) were used, whereas the inverted MHA structure was created using a negative-tone resist (TLOR-N001 PM, Tokyo Ohka Kogyo Co., Ltd.). The resist pattern was converted into metal structures by magnetron sputtering $50 \mathrm{~nm}$ of Pd on the resist pattern (AXXIS, JKLesker) and performing lift-off in an organic solvent of $\mathrm{CH}_{2} \mathrm{Cl}_{2}$, and then rinsing, in acetone and methanol.

The optical properties of NDA and MHA structures were measured using a Fourier transform infrared spectrometer (FT-IR 6200, JASCO Corporation). The sample chamber was evacuated using a rotary vacuum pump. Following evacuation, a $4 \%$ hydrogen/nitrogen gas mixture was injected into the sample chamber (volume $\sim 10000 \mathrm{~cm}^{3}$ ). The hydrogen flow rate was controlled using a mass flow meter and maintained at $1000 \mathrm{sccm}$. Optical transmission spectra were recorded over $5 \mathrm{~min}$.

\section{Results and Discussion}

To be suitable for plasmonic applications, a metal should preferably have a large negative $\varepsilon_{1}$ and $\varepsilon_{2}$ (i.e., a large negative dispersion factor $\varepsilon_{1} / \varepsilon_{2}$ ). However, contrary to conventional plasmonic materials, Pd has a fairly large $\varepsilon_{2}$ (shown in Fig. 1), which translates to significant optical losses at visible wavelengths. This issue with $\mathrm{Pd}$ can be mitigated by probing it in the mid-infrared. The optical permittivity of a metal can be described by the Drude model using the following equation: ${ }^{(13-16)}$

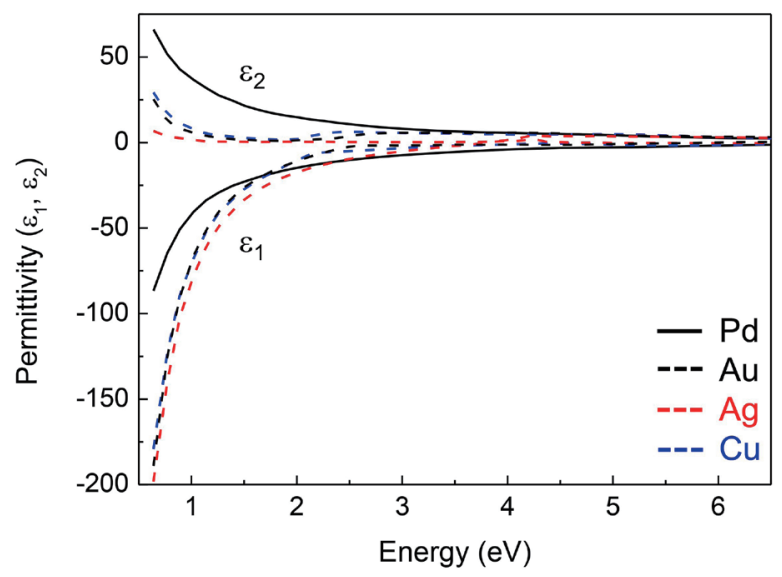

Fig. 1. (Color online) Optical permittivities of $\mathrm{Pd}$ and conventional plasmonic metals (Au, $\mathrm{Ag}$, and $\mathrm{Cu}$ ). The data is plotted according to Refs. 11 and 12. 


$$
\varepsilon(\omega)=\varepsilon_{\infty}-\frac{\omega_{P}^{2}}{\omega^{2}+1 / \tau^{2}}+i \frac{\omega_{P}^{2} / \tau}{\omega\left(\omega^{2}+1 / \tau^{2}\right)},
$$

where $\varepsilon_{\infty}$ is the permittivity at the high frequency limit, $\omega_{P}$ is the plasma frequency, and $\tau$ is the relaxation time. With this, metals can be characterized by performing least-squares curve fitting of experimentally measured permittivity to the Drude equation with $\varepsilon_{\infty}, \omega_{P}$, and $\tau$ set as free parameters. Drude parameters obtained in this way are listed in Table 1.

The permittivity parameter $\varepsilon_{\infty}$ is affected by the high-frequency tailing-off of the Lorenz oscillation. Ideal metals are expected to have $\varepsilon_{\infty}=1$. However, as a result of interband transitions, $\mathrm{Au}$ and $\mathrm{Cu}$ have a Lorenz oscillation in visible to near-infrared regions, whereas $\mathrm{Ag}$ and $\mathrm{Pd}$ stay close to the behavior of a pure Drude metal. The plasma resonance angular frequency $\omega_{P}$ is related to the ratio of the free electron density $N$ to the electron effective mass $m\left(\omega_{P} \propto \sqrt{(N / m)}\right)$. All of the aforementioned metals have comparatively similar values of these parameters (the density and effective masses fall in the range of $N, 10^{22}-10^{23} \mathrm{~cm}^{-3} ; m, \sim 1 m_{e} ; c f . N, \sim 10^{12} \mathrm{~cm}^{-2} ; m, \sim 0.07 m_{e}$ for graphenes; $N, 10^{16}-10^{18} \mathrm{~cm}^{3} ; m, 0.1 m_{e}-0.9 m_{e}$ for most semiconductors such as Si, GaAs, and InP). ${ }^{(17-21)}$ On the other hand, there is a significant disparity when it comes to the relaxation time $\tau$. For $\mathrm{Pd}$, this figure of merit is 9 times lower than that for $\mathrm{Au}$ and 30 times lower than that for Ag. Therefore, it is obvious that the strong damping of electrons in Pd results in the rapid decay of plasmonic oscillations; hence, achievable electromagnetic field enhancements are smaller than in conventional plasmonic materials.

Figure 2(a) shows scanning electron microscopy (SEM) images of NDA and MHA structures. Four sets of structures with different feature diameters and array periods were prepared by changing the scale of the projected exposure pattern. The mask was designed to have a period-todiameter ratio of 0.5 . This ratio shows the highest $Q$ factor in both NDA and MHA structures. ${ }^{(16)}$ As the evaluation from the SEM image, the real size of periodicity and disc/hole size were plotted in Fig. 2(b). NDAs were close to the designed size; however, MHA structures turned out smaller $(\sim 0.4)$ as a result of underexposure. The hole size does not affect the peak wavelength so much; this ratio is sufficiently effective for the hydrogen detection.

Figure 2(c) shows the optical transmission spectra of NDA and MHA structures. In the midinfrared region of interest, there is a multitude of background absorption bands resulting from $\mathrm{H}_{2} \mathrm{O}$ rotational modes $(1.8,2.7,5.2-7.6 \mu \mathrm{m})$ and $\mathrm{CO}_{2}$ vibrational modes $(\sim 4.3 \mu \mathrm{m})$ (shown in the upper part of Fig. 3). These absorption bands complicate the quantitative measurements of optical gas sensors in the mid-infrared and should be avoided. In this study, the plasmon resonance peak for our samples was tuned to be located between the $\mathrm{CO}_{2}$ and $\mathrm{H}_{2} \mathrm{O}$ absorption peaks. As a result, the periodicities of 1.41 and $1.48 \mu \mathrm{m}$ for NDA and 1.36 and $1.41 \mu \mathrm{m}$ for MHA could avoid the effect of $\mathrm{CO}_{2}$ and $\mathrm{H}_{2} \mathrm{O}$ absorptions.

Table 1

Drude parameters of Pd and conventional plasmonic metals.

\begin{tabular}{lccll}
\hline & $\mathrm{Au}$ & $\mathrm{Ag}$ & $\mathrm{Cu}$ & $\mathrm{Pd}$ \\
\hline$\varepsilon_{\infty}$ & 11 & 3.6 & 7.6 & 0.27 \\
$\omega_{P}\left(\times 10^{16} \mathrm{~s}^{-1}\right)$ & 1.4 & 1.4 & 1.3 & 1.3 \\
$\tau\left(\times 10^{-15} \mathrm{~s}\right)$ & 8.4 & 32 & 6.4 & 0.92 \\
\hline
\end{tabular}




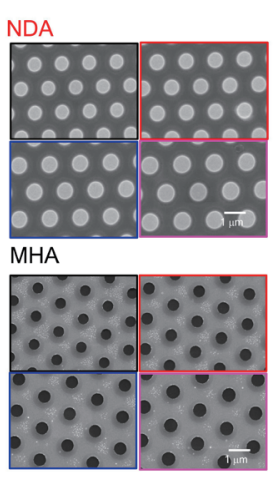

(a)

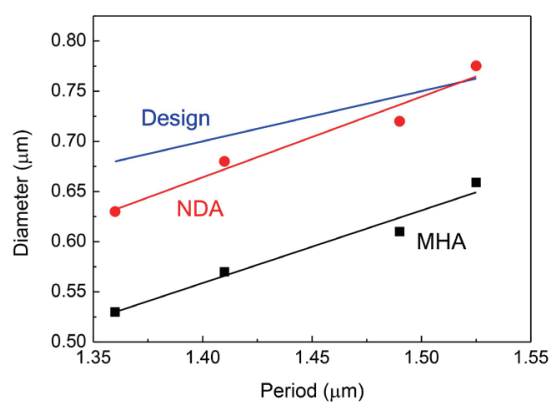

(b)

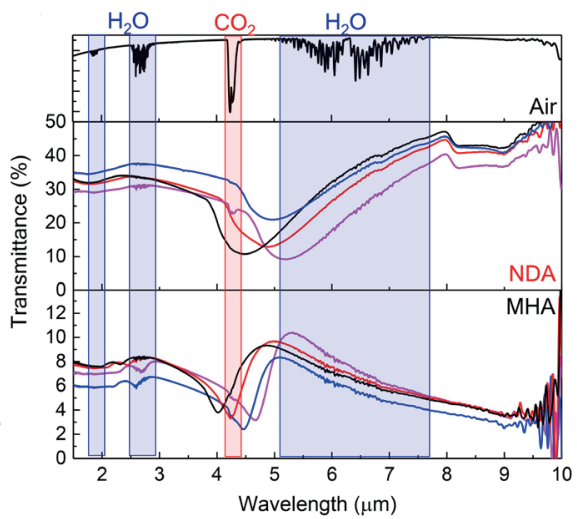

(c)

Fig. 2. (Color online) (a) SEM images of Pd NDAs and MHAs. (b) Relationship between array periods and feature diameters of fabricated NDAs and MHAs. The blue line represents the mask pattern as designed. (c) Optical transmittance spectra of air, NDA, and MHA structures.

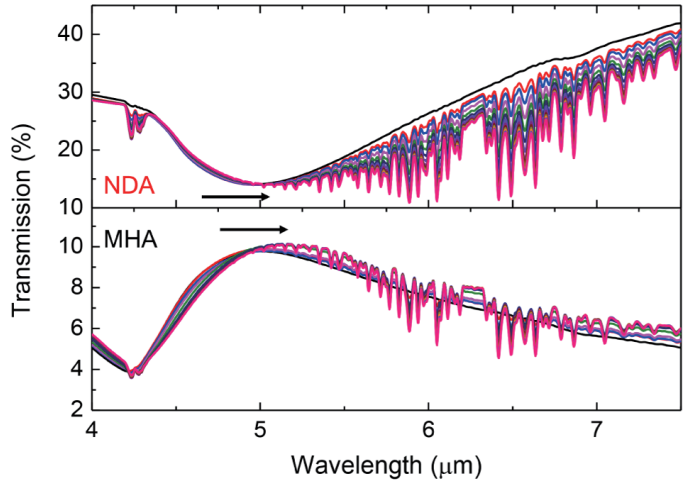

(a)

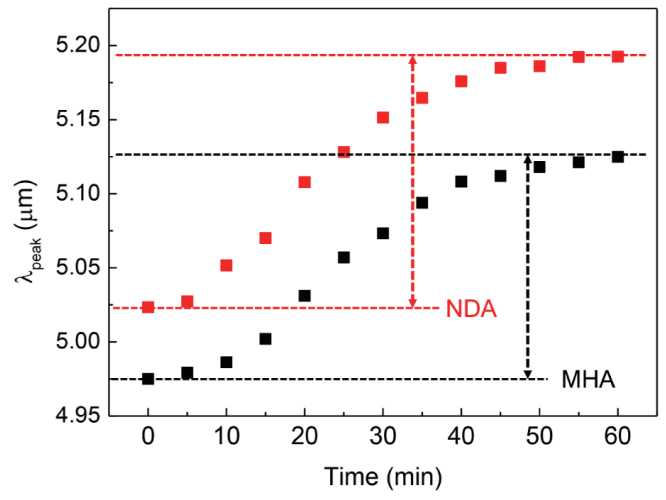

(b)

Fig. 3. (Color online) (a) Hydrogen exposure response for the NDA and MHA structures; spectra were measured every 5 min. (b) Time response of the transmission peak shift of NDA and MHA in 4\% hydrogen atmosphere.

NDA structures can be approximated as oblate spheroids described by the Mie scattering theory. According to the theory, plasmonic properties are linearly proportional to the disc diameter and thickness. Conversely, the optical properties of MHA are proportional to the periodicity of the hole array. The ratios of periods and diameters are almost constant; therefore, the plasmon peaks shift toward longer wavelengths as their diameters or periods increase.

Figure 3(a) shows spectral changes in the optical transmission of NDAs and MHAs as a result of exposure to hydrogen gas. In both cases, plasmonic spectral bands shift towards longer wavelengths. Hydrogenated Pd appears to exhibit dielectric properties; hence, the complex optical constants $(n=n+i k)$ show an increase in the real part $n$ and a decrease in the imaginary part $k$. However, $k$ decreases by a larger amount than $n$ increases; therefore, both $\varepsilon_{1}=n^{2}-k^{2}, \varepsilon_{2}=2 n k$ end up with lower values. The observed redshift of the plasmonic peak occurs as a result of changes in 
these optical constants. ${ }^{(22)}$ Moreover, the hydrogenated Pd feature has appeared on the plasmonic spectral width. Both NDA and MHA spectra were broadened with hydrogenation around $350 \mathrm{~nm}$. This is due to the effect that Pd decreases the metallic property owing to bonding with hydrogen.

With hydrogen exposure, a slight increase in the 5-7 $\mu \mathrm{m} \mathrm{H}_{2} \mathrm{O}$ related absorption lines was observed during measurements. This was due to a small leak of hydrogen oxidized at the thermal FT-IR radiation source; however, it did not disturb the overall analysis of broadband plasmonic spectral features.

Figure 3(b) shows the temporal response of the resonance peak shift during exposure to hydrogen. Both NDAs and MHAs exhibit a similar response. The spectral peak shift saturated after $40 \mathrm{~min}$ of hydrogen exposure, and a total change in wavelength of 140-160 nm was observed. This pattern was repeated for all of the samples with varying periodicities. A general advantage of mid-infrared plasmonics is that the overall observed resonance shift is larger than that in typical visible to near-infrared wavelength hydrogen sensors. On the other hand, in typical visible and near-infrared Pd nanoplasmonic hydrogen sensing, the response saturation time falls within a few minutes, whereas our results exhibited a slower rate. This is at least in part due to the chamber volume being large compared with the hydrogen flow rate. In general, the hydrogen intake process is limited by the surface reaction. The rate was affected by the surface area. It took more than 10 min to fill up 4\% hydrogen in the chamber. Therefore, the hydrogen supply is the rate-limiting process in this study. If uniform gas exposure can be set rapidly enough (for example, over a few seconds), a more definitive discussion on the manner by which size, volume, or structural differences between the plasmonic structures affect the time response to gas exposure could be offered.

\section{Conclusions}

In this study, we demonstrated Pd plasmonic nanostructures for all-optical hydrogen sensing at the mid-infrared wavelength for the first time. Both NDA and MHA show a similar response to the hydrogen exposure in spite of the different volume ratios and surface areas. This might be affected by the hydrogen response of Pd itself. The larger redshift of plasmon resonance in MIR wavelength region is useful for the stable hydrogen sensing.

\section{Acknowledgments}

The authors are grateful to the Tokyo Ohka Kogyo Co., Ltd. for their support of reduction exposure lithography. YN is grateful for partial support from the Japan Society for the Promotion of Science (JSPS), Grants-in-Aid for Scientic Research, Open Partnership Joint Projects of JSPS Bilateral Joint Research Projects, the Tateishi Foundation, and the Amada Foundation. SJ is grateful for partial support via the Australian Research Council DP130101205 Discovery project.

\section{References}

1 K. Ota, A. Ishihara, K. Matsuzawa, and S. Mitsushima: Electrochemistry 78 (2010) 970.

2 S. Masuzawa, S. Okazaki, and Y. M. T. Mizutani: Sens. Actuators, B 217 (2015) 151.

3 K. Tokutake and S. Okazaki: Sens. Actuators, B 191 (2014) 165.

4 E. Maeda, S. Mikuriya, K. Endo, I. Yamada, A. Suda, and J. J. Delaunay: Appl. Phys. Lett. 95 (2009) 133504. 
5 E. Maeda, T. Matsuki, I. Yamada, and J. J. Delaunay: J. Appl. Phys. 111 (2012) 084502.

6 A. Tittl, P. Mai, R. Taubert, D. Dregely, N. Liu, and H. Giessen: Nano Lett. 11 (2011) 4366.

7 C. Wadell, S. Syrenova, and C. Langhammer: ACS Nano 8 (2014) 11925.

8 N. Liu, M. L. Tang, M. Henrschel, H. Giessen, and A. P. Alivisatos: Nat. Mater. 10 (2011) 631.

9 T. Segai, P. Johansson, C. Langhammer, and M. Kall: Nano Lett. 12 (2012) 2464.

10 Y. Nishijima, S. Shimizu, K. Kurihara, Y. Hashimoto, H. Takahashi, A. Balčytis, G. Seniutinas, S. Okazaki, J. Juodkazyte, T. Iwasa, T. Taketsugu, Y. Tominaga, and S. Juodkazis: arXiv:1706.05517 (2017).

11 P. B. Johnson and R. W. Christy: Phys. Rev. B 6 (1972) 4370.

12 P. B. Johnson and R. W. Christy: Phys. Rev. B 9 (1974) 5056.

13 Y. Hashimoto, G. Seniutinas, A. Balčytis, S. Juodkazis, and Y. Nishijima: Sci. Reports 6 (2016) 25010.

14 Y. Nishijima and S. Akiyama: Opt. Mat. Express 2 (2012) 1226.

15 Y. Nishijima, Y. Hashimoto, G. Seniutinas, L. Rosa, and S. Juodkazis: Appl. Phys. A 117 (2014) 641.

16 Y. Nishijima, L. Rosa, and S. Juodkazis: Opt. Express 20 (2012) 11466.

17 L. Ju, B. Geng, J. Horng, C. Girit, M. Martin, Z. Hao, H. A. Bechtel, X. Liang, A. Zettl, Y. R. Shen, and F. Wang: Nat. Nanotech. 6 (2011) 630.

18 K. Zou, X. Hong, and J. Zhu: Phys. Rev. B 84 (2011) 085408.

19 L. E. Brus: J. Chem. Phys. 80 (1984) 4403.

20 R. T. Beach and R. W. Christy: Phys. Rev. B 16 (1977) 5277.

21 J. M. Luther, P. K. Jain, T. Ewers, and A. P. Alivisatos: Nat. Mater. 10 (2011) 361.

22 J. I. Avila, R. J. Matelon, R. Trabol, M. Favre, D. Lederman, U. G. Volkmann, and A. L. Cabrera: J. Appl. Phys. 107 (2010) 023504. 